\title{
El campamento educativo como escenario para el fortalecimiento de habilidades sociales The educational camp as a setting for strengthening social skills
}

\author{
Andrés Bal cázar Vega \\ Universidad de los Llanos (Colombia)
}

\begin{abstract}
Resumen. Este articulo condensa los resultados de las reflexiones generadas en torno a un proceso investigativo - aún en curso» que pretende determinar la maneraen quelos campamentos educativos contribuyen al fortal ecimiento de las habilidades social es. La investigación, adoptó la metodología estudio de caso. No obstante, los aspectos que motivaron esta publicación, se derivan de los debates del grupo de investigación, durantelarevisión bibliográficarequerida parala construcción del estado del arte, las categorías de análisis y la elaboración del marco conceptual del proyecto que, hasta la fecha, ha permitido reconocer las virtudes de loscampamentos educativos. En este sentido, es necesario contemplar la asertividad como unade las habilidades sociales a fortalecer en los campamentos educativos; iqualmente, es imprescindible la promoción de las directrices que regulan el comportamiento de esa micro-sociedad constituida en el campamento, la cual es pertinente para garantizar la convivencia pacífica de los acampantes. Identificando de acuerdo con las experiencias de campamento desarrolladas en municipio deVillavicencio - Colombia, los cuatro momentos o escenarios ideales de esta práctica, para poner en marcha el acto de educar, así como, las posibles acciones asumidas por los participantes, para generar cavilaciones conducentes al fortalecimiento de habilidades. Por último, las conclusiones reflejan la viabilidad de articular los campamentos a los diseños curriculares de las instituciones educativas.
\end{abstract}

Palabras claves: Campamento, habilidades sociales, comunicación, asertividad.

\begin{abstract}
This article condenses the results of the reflections generated around an investigative process - still ongoing - that seeks to determine the way in which educational camps improve the strengthening of social skills. The research adopted the case study methodology. However, the aspects that motivated this publication, are derived from the debates of the research group, during the bibliographic review required for the construction of the state of the art, the categories of analysis and the elaboration of the conceptual framework of the project that, to date, has all owed to recognizethe virtues of educational camps. In this sense, it is necessary to consider assertiveness as one of the social skills to be strengthened in educational camps; Likewise, it is essential to promote the guidelines that regulate the behavior of that micro-society constituted in the camp, which is per tinent to guarantee the peaceful coexistence of the campers. Identifying, according to the camping experiences developed in the municipality ofVillavicencio - Colombia, the four ideal moments or scenarios of this practice, to start the act of educating, as well as, the possible actionstaken by the participants, to generate conducive reflections to strengthening skills. Finally, the conclusions reflect the feasibility of articulating the camps to the curricular designs of educational institutions.

Keywords: Camp, social skills, communication, assertiveness.
\end{abstract}

\section{Introducción}

Los campamentos, en el ámbito internacional han sido abordados como objeto de estudio por diversas investigaciones, «Con el paso del tiempo, los campamentos se han modificado para responder a diferentes segmentos de mercado y a transformaciones socioculturales, lo que en gran medida ha permitido su diversificación» (Rosales, 2017, p. 11). En este sentido, surgen los campamentos educativos, develando que en el marco de una institucionalidad educativa se asuman como estra

Fecha recepción: 22-07-20. Fecha de aceptación: 30-11-20

Andrés Balcazar Vega

abalcazar@unillanos.edu.co tegias de intervención en el tiempo libre de niños y jóvenes, promotoras de transformaciones de convivencia entre compañeros, con el entorno (ambiental y cultural) y con uno mismo (Álvarez \& Verrastro, 2013). Sin embargo, en el contexto local, a pesar de que existen instituciones que promueven este tipo de prácticas no se conocen investigaciones desarrolladas respecto al tema.

Este artículo, deja ver un ejercicio de reflexión producido en el marco de un proceso de investigación «en ejecución» en el que se adoptó la metodología estudio de caso intrínseco, y con el cual se pretende determinar la relación entre las habilidades sociales y los campamentos educativos desarrollados en cuatro instituciones de carácter público del municipio deVillavicencio - 
Colombia. El escrito se ha organizado de manera lineal y progresiva iniciando con una aproximación de lo que son los campamentos educativos, sugiriendo dos premisas fundamentales a la hora de implementar este tipo de prácticas, para lo que acentúa la importancia de estimular el desarrollo de la habilidades sociales, mostrando lo necesario de una planificación oportuna, inmersa en su desarrollo; seguidamente devela la asertividad como una de las habilidades social es ideales para fortalecer en el contexto de campamento, deteniéndose en la comunicación verbal y no verbal.

Con el propósito de dar mayor claridad y poner en contexto el tema abordado, se propone la tabla uno en la que se refieren - de acuerdo con las prácticas actuales de campamentos educativos desarrollados en el municipio de Villavicencio - Colombia» los ambientes idea les para generar reflexiones en torno a las habilidades sociales, así, como las posiblesacciones de losacampantes de cara a esos momentos. Finalizando con conclusiones resultantes del proceso de reflexión generado por lo avanzado del ejercicio investigativo.

\section{Aproximacionesconceptualessobrecampa- mento educativo}

Al hablar de campamento es necesario reconocer que éste cuenta con una variedad de particularidades que se dan acorde a la intencionalidad con que se desa rrolle, en este orden se podrá encontrar campamentos organizados, educativos, organizados con propósitos educativos, escolares, acampadas, entre otros, no obstante, el énfasis que encontrará en este artículo obedece a los campamentos educativos.

Los campamentos educativos según Zipitría (2016) son un proceso organizado que se afirma sobre tres pilaresfundamental essopor tados desde la educación no formal, la dinámica grupal y la vida al aire libre, siendo imprescindibles para la fundamentación de valores en el desarrollo de la actividad. Lo cual permite que se catalogue como verdaderos laboratorios de convivencia, en losque el coordinador y su equipo directivo plantea una serie de directrices encaminadas a posibilitar el al cance de los objetivos propuestos para su desarrollo, pues son estos últimos, los que le otorgan la connota ción a este tipo de experiencias educativas desarrolla das al aire libre.

El campamento en un concepto muy general, puede ser definido como una actividad al aire libre que tiene un propósito determinado. Este fin puede ser utilitario, educativo, de supervivencia, de guerra 0 de interés so- cial, lógicamente para la Especialización en Educación para la Recreación Comunitaria, el campamento debe ser un campamento organizado y con carácter educativo que lleve a la comunidad respectiva a desarrollarse en el ámbito recreativo (Castañeda, 2011).

Es así, que para Álvarez y Verrastro (2013) el campamento educativo se identificacomo una actividad educativa desarrollada simultáneamente con la escuela moderna en ocasiones de manera autónoma y en otras complementando la enseñanza, ofreciendo diversas oportunidades educativas como la convivencia, la interacción con la natural eza, la asunción de roles y responsabilidades la resolución creativa de diversos desafíos etc.

Para lo cual, es necesario que cada campista se articule a esta dinámica, basándose en dos premisas funda mentales, «la unión hace la fuerza» y «la cadena se rompe por el eslabón más débil», respecto a la primera, se requiere tener en cuenta que para cualquier ejercicio colectivo que pretenda la optimización de tiempo y de esfuerzos, es fundamental la realización de un trabajo coordinado, en donde cada individuo conozca con exactitud cuál es su compromiso y el rol a desempeñar, al igual que sus límites; de manera, que desde su responsabilidad individual tenga presente, que sus acciones son imprescindibles para el beneficio de todo el grupo, aunque en ocasiones sienta, que está trabajando más para la comodidad de los otros que para la suya.

Es por esto que para Mendoza (2017) el trabajo colaborativo se asocia con la metodol ogía de aprendiza je basado en problemas permitiendo que los alumnos adquieran competencias para la vida laboral ya que las interacciones les facilitan dar y recibir información sobre la construcción de soluciones a situaciones problémicas.

Con el pasar del tiempo en el campamento se develará la reciprocidad del asunto, descubriendo que sus aportes han logrado beneficiar al resto de los campistas, así como la cooperación de los otros, le han generado grandes beneficios a él.

En este sentido, el campamento permite experimentar aquellos adagios populares como: el todo es más que la suma de sus partes, juntos somos más fuertes, la inteligencia grupal, supera la inteligencia individual, etc. Invitando a tener presente que vale la pena esforzarse por el bienestar de los demás, y a valorar el esfuerzo que hacen los demás, para generar comodida des que también le favorecerán.

Frente a la segunda premisa, es importante que el coordinador (como campista mayor) y el equipo orga nizador del campamento, tengan conocimiento de al- 
gunos patrones comportamentales de cada uno de los acampantes, por ejemplo, sobre rasgos de su personal idad que les facilite tener presente el temperamento, timidez, amabilidad, agresividad, introversión, extra versión, resiliencia, machismo, feminismo, pereza, intrepidez, entre otros; esto, le será muy útil en el momento de conformar los equipos (bosques o patrullas) de trabajo, buscando su heterogeneidad «para promover la inclusión» de manera que ninguno quede recargado de al gún grupo de personas temperamentales 0 en su defecto, muy pasivas o con comportamientos particulares; en procura, de neutralizar a quienes suelen ser más agresivos, motivar a los más pasivos y tener una estrategia pedagógica para garantizar la participación de todos en la experiencia de acampar, independientemente de su personalidad.

En este orden de ideas, y continuando con la analogía de la cadena; el eslabón más débil, pude ser origina do por la intolerancia de un campista frente a la actitud pasiva de otro, de la actitud introvertida de uno, frente a la personalidad extrovertida de otro, del temor de uno respecto a la intrepidez de otro o frente a la falta de resiliencia de alguno entre muchas otras posibilidades. Por lo tanto, al permitir que una de estas conductas se desborde en el campamento, se corre el riesgo de generar tensiones en la convivencia de todo el grupo, es por esto, que «La conducta de la población participante esun temaque preocupaatodas las personas involucradas en la actividad de campamentos y cual quier otra actividad» (M ejía et al., 2009, p. 192).

De esta manera, un manejo inapropiado de una situación derivada de comportamientos indebidos, puede desencadenar un desafortunado efecto dominó, involucrando cada vez más acampantes y agravando el problema, es por eso que parte de las tareas de la directiva del campamento, es mantenerse muy atentos a todo aquello que pueda debilitar uno de los eslabones de la mencionada cadena, para así, oportunamente darle el manejo apropiado y evitar episodios que puedan atentar con el desarrollo del campamento.

De esta forma, se debe convertir en regla de oro que entre los amigos de colegio se fomenten las habilidades sociales para lograr mantener los propósitos de este tipo de prácticas, donde mediante el desarrollo de dichas habilidades se puede fortalecer el compañerismo tal como lo asumen Rodríguez, Hernandez y Triana (2019) quienes expresan que «el compañerismo deja ver diferentes modelos de amistad en el que se hallan diferencias entre sí, sin embargo, difiere de la amistad por el contexto social en el que se desenvuelve» (p.52).
Siendo esta una de las razones por las cuales se puede afirmar que:

Los campamentos se han destacado como una estrategia privilegiada de inter vención en el tiempo libre de niños y jóvenes, donde se generan procesos transforma dores de convivencia con otros compañeros, con el entorno (ambiental y cultural) y con uno mismo. Se la considera una herramienta fundamental en el desarroIlo integral de la persona, ya que promueve el desarroIlo de la afectividad, la creatividad, la sociabilidad, la espontaneidad, la autonomía personal y de habilidades sociales y éticas (Gómez, Gonnet \& Lema, 2017).

Sin embargo, se considera importantetener en cuenta que la práctica de campamento per se, -es decir, sin ningún tipo de planeación, o propósito educativo establecido- no gar antiza que se genere al guno de los aspectos citados anteriormente.

\section{Dirección en los campamentos}

Es completamente necesario mantener un escalafón que le dé cabida legitima a la capacidad de dirigir, evitando que en el campamento se establezca una anarquía, que conduzca a los campistas a hacer desde la individualidad lo que consideren importante, en el momento que lo crean necesario, ocasionando grandes dificulta des para dar cumplimiento a un cronograma de actividades y entorpeciendo el alcance de cualquier objetivo de carácter educativo.

Lo anterior, hace imperativo que siempre que se planifique la estructuración metodológica de un ejercicio de campamento se tenga presente que «La masificación deloscampamentosy su consolidación como propuesta educativa institucional izada, plantea dos desafíos a resolver: la necesidad de diseñar los campamentos como procesos pedagógicos y la necesidad de prepa rar a los educadores para intervenir en campamentos» (Álvarez \& Verrastro, 2013, p 32).

Sin embargo, esto no significa que no se puedan rea lizar campamentos sin tener en cuenta unos rangos preestablecidos entre sus acampantes, lo que sucede es que en estos casos, solo se hacen con fines recreativos y en grupospequeños (familiareso amigos), donde finalmente afloran cier tos liderazgos innatos en al gunos de sus participantes, que influyen en la toma de decisiones, como para determinar la preparación de los alimentos, la recolección de leña, el aseo del campamento, el manejo de los residuos, la hora de dormir, etc.

Así también, es importante develar de donde surgen las directrices del campamento y quienes son los 
responsables de promover su cumplimiento, de manera que el docente o coordinador no sea el único que visibilice su rango superior al impartir directrices, cual si estuviese a la cabeza de un régimen dictatorial; sino, hacer de este escenario una oportunidad para que en el marco de un ejercicio democrático, surjan nuevos dirigentes juveniles que gracias a sus potencialidades sean elegidos por sus compañeros (al interior de cada grupo) para hacer parte de la coordinación del campamento.

Lo anterior, entendiendo que según el pensamiento de Alejandro (2013) la democracia necesita de la promoción de oportunidades equitativas frente a los asuntos públicos y en los procesos electorales garantizando inclusión, equidad e imparcialidad, por lo que el docente a cargo, no solo les otorgará ciertas funciones rela cionadas con la coordinación, la disciplina, el aseo, las técnicas de campamento (construcciones de cocina, mal etero, zapatero, tabúes, etc.) sino, que debe reunirse permanentemente con ellos para hacerlos participes de la tomade decisiones; teniendo claro que tal como lo plantea Castañeda (2011) la misión del coordinador es orientar un procesos de interacción en el que se estimulen las cualidades individual es y de grupo, así como el contribuir en el control de actitudes antisociales.

De esta manera se puede garantizar que el campa mento se desarrolle mediante un proceso dinamizador y participativo, al tenerse en cuenta no solo la opinión del docente 0 coordinador general, sino la de cualquier campista, la cual se canalizará más fácilmente a través de los dirigentes (por su condición de pares), que en línea directa de un estudiante campista, al docente coordinador; ya que no todos encuentran facilidad al dirigirse a una autoridad. Es por esto que también se hace favorableel egir entrelos estudiantescampistas, un coordinador general del campamento quien será asistido permanentemente por el docente a cargo o principal responsable de esta práctica.

Para que los dirigentes de los campamentos educativos puedan lograr este proceso dinamizador del programa, necesitan una identificación y motivación con los finesy los objetivos del Campamento, condición fundamental. En otras palabras, además de su preparación técnica específica en un área temática determinada, el liderazgo debe proyectar el gusto y el disfrute personal por aquellos temas y valores que el Campamento desea promover entre los campistas; esto de manera transversal durante toda la ejecución del programa (Ferrer \& Sánchez, 2011).

Así también, la experiencia de quienes asuman la dirección del campamento será mucho más enriquece- dora y formativa, ya que su nivel de compromiso y de responsabilidad aumenta respecto al resto de campistas y por otro lado, el hecho de que al guien que se encuentre en su rango de edad, nivel académico, estrato sociocultural o cual quier otro aspecto con el que se identifique, sea parte del grupo de quienes expiden las directrices y trabajan sobre la toma de decisiones; genera una gran enseñanza a los demás campistas, demostrando que las normas de convivencia, las tareas asignadas y todos los parámetros del campamento, pueden cumplirse en armonía, sin que necesariamente tenga que haber un profesor 0 autoridad académica al frente de cada proceso.

Mostrando de esta manera la capacidad de dirigir, junto a las habilidades para trabajar en equipo, como un conjunto de competencias a desarrollar en los acampantes. «0 tro conjunto de competencias apuntan al desarrollo de la dimensión social: sociabilidad, interrelación, compañerismo y amistad, respeto al prójimo, convivencia, aceptación y reconocimiento social, ajuste social, hábitos adecuados de participación, situarse mejor con quienes le rodean, de responsabilidad cívica» (Gómez, Gonnet \& Lema, 2017, p 30). Además de todo aquello que promueve el espíritu de servicio y la participación social y comunitaria.

Cabe resaltar, que la estructura orgánica del campa mento, no solo ayuda al funcionamiento de este, respecto al manejo de la disciplina y al desarrollo de las actividades propias, sino que contribuye generosamente, con el fomento de habilidades sociales, las cuales, son necesarias en cada momento de la vida, especialmente cuando se requiere trabajar para alcanzar objetivos en común. Siendo el campamento un espacio en donde los participantes deben permanentemente aunar esfuerzos para el desarrollo de las labores diarias, viene bien que las relaciones interpersonales fluyan en un ambiente de confraternidad, dando garantía al bienestar emocional de cada ejercicio, entendiendo que «La necesidad de compartir entre estudiantes es particularmente relevante para los jóvenes pues se encuentran en una etapa en que sus pares constituyen el principal agente socializador.» (Tapia, Paz \& Cubo, 2017, p. 145).

Por otro lado, es sensato mencionar que aunque en Colombia la promoción de los campamentos educativos no se ofrece únicamente desde las instituciones educativas, sino también de entidades de carácter gubernamental, por ejemplo el Ministerio del Deporte viene ofreciendo el programa Campamentos Juveniles, el cual entre sus objetivos generales expresa su interés por «Fomentar la confraternidad entre los jóvenes colom- 
bianos, a través, de prácticas de campismo que estimulen el desarrollo de la personalidad y el afianzamiento de valores y conceptos.» (COLDEPORTES, 2015, p. 16). Es de valorar, que las instituciones escolares incorporen este tipo de prácticas con el fin de complementar sus estrategias educativas.

\section{Las habilidades sociales desde las prácticas de campamento educativo}

La compleja sociedad en que vivimos requiere una formación escolar que trascienda el simple aprendiza jes de contenidos académicos (Vivas et al., 2017) razón por la cual es necesario comprender que las habilidades sociales, como competencia a fortalecer en los acampantes, deben convertirse en su principal riqueza, a pesar de que, en la actual idad, no existe un consenso generalizado sobre la total idad de las habilidades socia les que podría comprender este constructo (Caballo \& Salazar, 2017). Tampoco una jerarquización definida de las mismas, es razonable que la comunicación asertiva y demás elementos que promueven este tipo de conductas socialmente habilidosas, se privilegien, gestionen y fomenten por la directiva del campamento, pues la responsabilidad principal de mantener un clima organizacional apropiado recae, en primer lugar, sobre ellay en segundo lugar sobre cadauno de losacampantes.

Cabe mencionar, que la conducta socialmente habilidosa es ese conjunto de conductas emitidas por un individuo en un contexto interpersonal, que devela los sentimientos, deseos, opiniones, actitudes de un modo armónico, que facilita la reducción de problemas futuros (Ramos, Cantillo \& Bestard, 2014).

Es de considerar que la atmosfera social de un campamento se da de acuerdo con las habilidades sociales que se gestionen durante el desarrollo de cada momento de actividad o de descanso, pues, a diferencia de las conductas o comportamiento aflorados en las jornadas escolares dentro de las instituciones educativas, 0 en el entorno habitual de los participantes; el campamento, exhorta alos acampantes a mostrar sus diferentes facetas comportamentales de manera permanente, ya que su actividad social, no se reduce a una jornada estudiantil, deportiva, recreativa o cultural como es habitual, sino que se revela las veinticuatro horas del día, durante todo el tiempo de acampada, dando lugar a que cada individuo paulatinamente se muestre tal como es, en momentos de trabajo, descanso, juego, de adversidad, y hasta mientras duerme.

Siguiendo con la idea anterior, el campamento es el escenario por excelencia para permitir que alguien interactúen con otros de la mejor forma, pues difícilmente se podrá conocer a una persona en un contexto diferente al acostumbrado compartiendo ratos de felicidad y momentos después, poniendo a prueba sus emociones al afrontar alguna adversidad, posteriormente saber que se está desvelando mientras presta guardia (turno de vigilancia), para luego escuchar hasta sus ronquidos durante el sueño, y al amanecer verla levantarse despelucada y sin acicalarse, esperando que llegue la hora del aseo personal.

Lo anterior, teniendo en cuenta que cada experiencia durante la acampada, permite que el acampante revele más fácilmente algunas de sus emociones, las cuales dependiendo de sus habilidades sociales pueden ser favorables o desfavorables para la convivencia del campamento, ya que no todos los acampantes han desarrollado los mismos niveles de tolerancia y la capacidad de controlar sus estados emocionales ante situaciones ambientales. como calor o frio extremo, presencia perma nente de insectos, humedad, Iluvia; u otras, concernientes a las relaciones interpersonales como por ejemplo, enojarse de manera exagerada por una caída al impactar con un compañero durante un juego motor, no soportar una broma y responder con agresividad, burlarse de algún compañero para ridiculizarlo, no respetar la condición de líder de al gún par, sentirse excluido de manera constante por el resto del grupo sin razón al guna, o tratar de excluir a otro por creerlo inferir a las necesidades del grupo, así como no estar atento a las normas de convivencia establecidas, entre otras.

En este sentido, un campamento se puede comparar con una pequeña micro-sociedad en donde fluyen diferentes formas de coexistir, de valorar, de asumir compromisos, de corresponderlea lanatural eza, espor esto, que desde la mirada de un educador, se convierte en el escenario perfecto para emprender procesos educativos especialmente relacionados con los patrones comportamentales delos participantes, razón por lacual, proponer esta práctica como un ejercicio que estimula el desarrollo de habilidades sociales, es completamente acertado; siempre y cuando, los objetivos de la misma proyecten su intencional idad haciala generación de aportes significativos para la educación integral de los participantes.

El campamento por sí solo, es apenas un espacio abierto donde pernocta un grupo de personas por un periodo de tiempo, atendiendo unas normas básicas de convivencia. Lo que marca la diferencia generándole una característica particular a este tipo de prácticas, es 
la intencionalidad con la que se realizan; pues esta, le otorgaunaconnotación o temáticay adicional mente abre el espacio para establecer un propósito, por ejemplo: campamento deportivo (como tema), sensibilizar hacia la iniciación deportiva (como propósito); campamento cultural (como tema), exposiciones de interpretación de instrumentos musicales (como propósito); campa mento educativo (como tema), fortalecimiento de ha bilidades sociales (como propósito).

Cabe agregar, que, así como de la práctica de campamento surge infinidad de temas a desarrollar, es necesario tener en cuenta, que de un tema de campamento pueden surgir un sinnúmero de propósitos; lo importante es definir muy bien el asunto, en sintonía con lo que se desea lograr.

Es así, que mediante la implementación de estrategias que contribuyan al fortal ecimiento de las habilidades sociales, como intencionalidad de un campamento escolar, se pueden generar un proceso de sensibilización o estimulación de dichas habilidades, siendo estas, uno de los componentes más importantes en el desa rrollo integral del ser humano, reconociendo que muchas investigaciones dejen ver cómo las competencias personales son mejor valoradas por los alumnos, seguida de las competencias sistémicas e instrumentales, destacando las competencias para el trabajo en equipo, habilidades interpersonales, criticas, trabajo interdisciplinar, etc (Pegalajar, 2018).

A pesar, de que, para un sistema educativo estructurado a partir de competencias, como el que rige en este país (Colombia) « al parecer, generador de grandes privilegios hacia el desarrollo cognitivo» que deja marginados otros aspectos del proceso formativo, como los relacionados con las habilidades motrices, artísticas, y axiológicas; desestimando junto a estas, el valor de las habilidades sociales.

Por lo tanto, diseñar estrategias que promuevan las habilidades sociales debe convertirse en un compromiso de todo educador y al referirnos a estas, no se puede pasar por al to que las maneras de pensar y actuar de los infantes y jóvenes son influenciadas por sus metas, siendo estas una fuente motivacional que el profesional 0 padre debe conocer, ya que pueden ser adecuadas influyendo positivamente en el comportamiento interpersonal. Al no ser adecuadas, es necesario determinar por quéla persona afrontala actividad social como lo hace, para ayudarla a modificar dichas metas si es necesario (Pérez, 2000).

Entendiendo que, naturalmente existen muchos estudiantes que, durante las actividades académicas dia rias en sus instituciones, limitan sus relaciones sociales a pequeños grupos, sin importar que transcurran semanas, meses 0 años, sin ampliar el círculo de amigos que han constituido; tanto, que no es difícil encontrar un par de personas que estudiaron juntos todo el bachillerato y nunca compartieron una conversación por más de un par de minutos. Situación que corrobora, que los intereses académicos de algunas instituciones educativas, desestiman la importancia de las habilidades sociales en la formación ciudadana, siendo esto último para Sáez, Figueroa y Pereira (2018) un pilar fundamental de los procesos de enseñanza y aprendizaje de los estudiantes, ya que considera las competencias necesarias para armonizarse con una sociedad plural y axiológica

Lo anterior, obliga a los educadores a reflexionar frente a su compromiso de educar ciudadanos integra les, para lo cual, es necesario replantear algunas de sus prácticas, siendo el campamento escolar una de las experiencias que, desarrolladas de cara a la educación, puede contribuir con espacios de socialización, fomento de la pluralidad argumentada, desarrollo de la capacidad crítica, creatividad, trabajo en equipo, sensibiliza ción ambiental, resiliencia, etc. todo esto, iniciando con la ampliación de los círculos sociales de cada estudiante y además, atendiendo lo planteado por M oyano, Pacheco y U rbieta (2018) quienes sostienen que una actitud motivada por tareas centradas en la mejora personal, el progreso y la cooperación contribuye la obtención de estados psicológicos óptimos para el emprendimiento de actividades.

Lo que hace necesario, que el docente o coordina dor del campamento, quien entre sus funciones debe garantizar que los demás campistas articulen esfuerzos para trabajar por el alcance de los objetivos trazados, asegurando la organización de los equipos (bosques 0 patrullas) de trabajo, de manera que sean heterogéneos y equilibrados, es decir, que, si en el equipo hay un estudiante hábil motrizmente, los demás equipos cuenten con la misma particularidad. Además, es imprescindible que estos, no se constituyan por estudiantes camara das, preferiblemente, de aquellos que muy poco comparten experiencias en su cotidianidad.

De esta manera las habilidades sociales se ponen en contexto durante los procesos comunicacionales efectuados, no obstante, su naturaleza permite la posibilidad de que sean entrenadas para mejorar sus resulta dos; al igual que se entrenan las habilidades físicas en un escenario deportivo, el ambiente de campamento, es ideal para ponerlas a prueba, evaluarlas y potenciarlas; quienes han tenido algunas experiencias, dirigiendo 0 
participando en campamentos, saben que durante el mismo, tienden a develarse algunas actitudes relacionadas con las estructuras de aprendizaje; es decir habrán quienes tiendan a asumir los compromisos desde la individualidad, así como otros que lo hagan pretendiendo ser los primeros o los mejores adoptando actitudes competitivas y al gunos que prefieran naturalmente privilegiar el bien común, asumiendo comportamientos más solidarios y cooperativos.

Con el fin de darle contexto a lo anteriormente expuesto, esnecesario comprender que basadosen loscampamentos desarrollados por las instituciones educativas del municipio de Villavicencio, pueden existir cuatro momentos (ambientes) estratégicamente apropiados, para intervenir oportunamente con mayor facilidad mediante ejercicios educativos, con el propósito de estimular el fortalecimiento de estas habilidades sociales, dichos momentos y su relación con las posibles acciones se presentan en la tabla 1.

El cuadro anterior deja ver los cuatro momentos más relevantes que se pueden encontrar en un campa mento para reconocer los comportamientos de los campistas a la luz de las tres posibles acciones señaladas, que podrían ser de gran ayuda para la convivencia o por el contrario, un factor de riesgo para la misma, teniendo presente lo expuesto porVila et al. (2019) quien sostienen que «Los sujetos más cooperativos tienden a ser menos competitivos e individual istas mientras que los más individualistas suelen ser más competitivos». ( $p$ 105). Lo que indica que las posibles situaciones protagonizadas por al gunos de los acampantes, se pueden convertir en oportunidades para que el coordinador o líder analice las particularidades de las situaciones y basados en sus resultados, genere espacios de reflexión en los que se les escuche a los involucrados con la mayor serenidad y atención posible, promoviendo asertividad en la comunicación; ya que son este tipo de episodios, los que no se deben dejar pasar por alto para poner en mar- cha con gran agudeza el acto de educar.

No obstante, el coordinador no solo debe identificar los momentos de crisis, para promover espacios de reflexión, también lo puede hacer exaltando un comportamiento ejemplar que sirva como referente para los objetivos que se pretenden alcanzar con el campamento, sin olvidar que, al reconocer las virtudes educativas presentes en un campamento educativo, organizado y desarrollado con tales propósitos, es necesario gestionar permanentemente una comunicación asertiva, la cual compromete no solamente el lenguaje verbal, sino el lenguaje corporal, sin llegar a desconocer que «Aunque conscientemente nuestra atención parece centrarse en las palabras que constituirían el mensaje verbal, inconscientemente recibimos también la información no verbal, y nuestras impresiones se basan en los dos tipos de comunicación, siendo esta última de gran importancia» (Sánchez, 2016, p 54).

\section{La comunicación asertiva durante los cam- pamentos}

Definitivamente al pretender fomentar las habilidades sociales se hace completamente imprescindible el uso de una comunicación clara que no dé lugar a interpretaciones desafortunadas, pues una palabra inapropiada, el tono con que se hable, los ademanes que se utilicen, los gestos expresados al escuchar, etc. Son al gunos de los elementos que se deben empezar a regular, si se desea mejorar dichas habilidades; siendo en este punto, donde es necesario comprender con más detalle el concepto de asertividad, para lo que es pertinente mencionar que «Ciertos autores consideran la asertividad como la conducta que posibilita la disminución de la ansiedad, otros la consideran como la defensa de los derechos propios y al gunos como la habilidad para expresar sentimientos y pensamientos» (Pereira, 2008, p.3). Sin embargo, es preciso tener en cuentael siguiente

Tabla 1

Ambientes ideales para estimular habilidades sociales en campamentos educativos de acuerdo a experiencias en el municipio deVillavicencio - Colombia.

\begin{tabular}{|c|c|c|c|c|}
\hline Posibles acciones & Durante la aplicación de técnicas & En las labores de cocina & Durante las actividades de la fogata & En el desarrollo de los juegos \\
\hline Individuales & $\begin{array}{l}\text { El campista se interesa exclusivamente por } \\
\text { organizar su carpa (tienda), y demás el ementos } \\
\text { que garanticen su comodidad per sonal, } \\
\text { desentendiéndose de la cocina, la letrina, el } \\
\text { tabú y otros elementos de bienestar común. }\end{array}$ & $\begin{array}{l}\text { El campista tiende a preparar los } \\
\text { salimentos teniendo en cuenta sus gustos } \\
\text { personales, desconociendo de alguna } \\
\text { manera los de la mayoría de compañeros. }\end{array}$ & $\begin{array}{l}\text { Trata de motivar a los otros acampantes a real izar } \\
\text { actividades que personalmente le agraden, } \\
\text { ejemplo: persuade a los demás para contar chistes, } \\
\text { así entienda que lo que desean es cantar. }\end{array}$ & $\begin{array}{l}\text { Se interesa más por vincularse en juegos que } \\
\text { requieren pocos participantes y al terminar se } \\
\text { siente más cómodo iniciando de nuevo que } \\
\text { cediendo el turno a otro. }\end{array}$ \\
\hline Competitivas & $\begin{array}{l}\text { El campista prefiere realizar sus construcciones } \\
\text { de manera aislada, sin compartir } \\
\text { conocimientos respecto a la elaboración de } \\
\text { nudos o amar res, procurando rapidez y calidad } \\
\text { para demostrar que es mejor que los demás. }\end{array}$ & $\begin{array}{l}\text { sSe preocupa por ser el más rápido por } \\
\text { ejemplo pelando papas, prendiendo el } \\
\text { fogón etc. De igual forma, por preparar } \\
\text { los alimentos de la manera más apetitosa } \\
\text { posible, no para que los demás coman con } \\
\text { agrado, sino para ser admirado por sus } \\
\text { habilidades en cocina. }\end{array}$ & $\begin{array}{l}\text { Se interesa por organizar concursos en los que sus } \\
\text { habilidades artísticas sean favorecidas; es decir, que } \\
\text { si es bueno cantando, no le será suficiente cantar, } \\
\text { sino participar en un concur so de canto, para así } \\
\text { hacer ver que es el mejor. }\end{array}$ & $\begin{array}{l}\text { No siente mayor gusto por juegos de } \\
\text { integración o cooperativas. Considera que la } \\
\text { esencia de un campamento radica en el } \\
\text { desarrollo de juegos tipo desafío, razón por la } \\
\text { cual es el mayor promotor de esta clase de } \\
\text { actividades. En las que, en muchas ocasiones } \\
\text { son intolerantes ante los fracasos. }\end{array}$ \\
\hline Cooperativas & $\begin{array}{l}\text { Se esmera por compartir sus conocimientos en } \\
\text { cuanto a técnicas de campamento, explicando a } \\
\text { sus compañeros lo que este a su al cance para } \\
\text { garantizar que todos se sientan a gusto en la } \\
\text { construcción del campamento. }\end{array}$ & $\begin{array}{l}\text { Genera ar monía entre los que saben } \\
\text { acocinar y los que no, asumiendo rol de } \\
\text { asistente del que sabe, o en su defecto, } \\
\text { motivando al que no sabe a realizar } \\
\text { actividades como lavar papas, pelar } \\
\text { plátanos picar cebolla etc. }\end{array}$ & $\begin{array}{l}\text { Motiva a sus compañeros a participar en las } \\
\text { actividades (cuentería, teatro, performans, rondas, } \\
\text { ritos, chistes, mitos y leyendas etc.) ), promoviendo } \\
\text { el respeto, y dando tranquilidad a aquellos que } \\
\text { teman ser ridiculizados. }\end{array}$ & $\begin{array}{l}\text { Al participar en juegos tipo desafío, posibilita la } \\
\text { interacción promotora entre los miembros de } \\
\text { su equipo, se ar moniza con todos los } \\
\text { integrantes, es tolerante frente a las der rotas y } \\
\text { prudente respecto a las victorias. }\end{array}$ \\
\hline
\end{tabular}


concepto de asertividad, el cual «hace referencia a las habilidades interpersonales que implican la expresión directa de la defensa de los propios derechos y opiniones personales sin negar los derechos y la opinión de los otros, el autorefuerzo y el refuerzo a los demás (Pérez, 2000, p. 39).

Ahora bien, teniendo en cuenta las reflexiones afloradas por el autor, las cuales surgen desde la experiencia de los campamentos escolares realizados en el municipio de Villavicencio - Colombia, en los que ha participado y del ejercicio investigativo que motivó la elaboración de este artículo, cabe mencionar que para promover la asertividad durante este tipo de prácticas, se requiere el fortalecimiento de cada uno de sus componentes, sin embargo, aquí se señalará la comunica ción por considerarse relevante; de la cual se deriva el lenguaje verbal y no verbal. Frente al lenguaje verbal se exponen al gunas consideraciones con el ánimo de que sean promovidas durante las prácticas de campamento:

Tono de voz: el tono de voz utilizado a la hora de mantener una conversación con una o más personas, debe ser no muy bajo que refleje debilidad, ni muy fuerte que refleje arrogancia. Lo ideal es que sea firme y bien acentuado.

- La velocidad del habla: evitar hablar con ligereza para alejarse de la taquilalia, ya que puede tornarse asfixiante en la conversación; tampoco hacerlo tan lento, ya que suele generar ansiedad en los interlocutores, quienes anhelarán la hora en que termine de expresar su idea.

Vocalización: es importante que la expresión oral se realice con muy buena vocalización, de esta manera se otorgará mayor facilidad y comodidad a quien escucha, evitando que este deba interrumpir para solicitar que se repita algún fragmento de lo ya expresado.

U so de muletillas: es más agradable escuchar un discurso, o algún tipo de intervención, cuando quien se expresa, evita caer en el uso de muletillas, ya que éstas, pueden generar distracción en quienes escuchan, así como la perdida de interés por lo que se está escuchando.

Capacidad de síntesis: procurar sintetizar la información de lo que se desea compartir, puede generar muchas ventajas, especialmente para garantizar la atención de quienes escuchan, esto permite que se optimice el tiempo de conversación (sí, es el caso) logrando que el intercambio de información fluya de manera más eficiente.

Léxico: hacer uso de un vocabulario acorde al contexto y al rol que se desempeñe es fundamental para armonizar el acto de comunicar, evitando ser tan refinado o preciosista, pero tampoco rayar en lo coloquial. De esta forma, será más fácil hacerse entender, y al mismo tiempo cautivar la escucha de los demás. Por otro lado, respecto al lenguaje (no verbal) corporal utilizado para acompañar las ideas que se desean compartir en el momento de conversar o dirigirse una persona o grupo de personas se sugiere tener en cuenta los siguientes aspectos:

Proxémica: no se pretende hacer mención a la proxémia como disciplina, sino más bien a su objeto de estudio, ya que se enfoca en todo lo que tiene que ver con el uso del espacio personal y las distancias sociales durante los procesos de comunicación verbal. Es decir, mantener una distancia prudente ante su interlocutor, no muy lejos que deba gritar, ni muy cerca que invada su intimidad. Se sugiere una distancia entre $1.30 \mathrm{M}$ ts a $45 \mathrm{cms}$ (durante comunicación personal), y de $3 \mathrm{M}$ ts a 3.50 M ts (durante comunicación social).

- Cronémica: a lo largo de la historia, se ha estructurado y culturalmente regulado, el uso de lostiempos en los momentos de intervención, cuando se participa de una conversación entre dos o más personas; esto se hace de manera inconsciente y posibilita que la comunicación fluya de manera bidireccional 0 multidireccional, dando posibilidad a que los interlocutores estén en igualdad de condiciones, respecto al tiempo para el uso de la palabra.

Postura: involuntariamente la postura expresa el deseo de continuar, o por el contrario, de terminar una conversación de la persona que habla, como de quien 0 quienes escuchan, por tal razón, es imprescindible que la actitud corporal expresada mediante la postura transmita un mensaje de disposición durante el ejercicio comunicacional.

- Expresión facial: los gestos faciales hablan por sí solos, y transmiten con una velocidad inimaginable lo que se está pensando, desde antes de decirlo, así como también la idea que se está asimilando, desde el momento en que empieza a ser escuchada, lo que hace que, en ocasiones, termine prestándosele mayor importancia a las expresiones faciales que a las verbales, ya que las primeras suelen ser más sinceras.

Asentir y sonreír: es señal de buena disposición, asentir, para demostrar a quien habla, que se está aprobando la idea expresada y si este acto se acompaña de una sonrisa, básicamente ratifica que no solo aprueba, sino que se identifica completamente con lo dicho; por otro lado, quien habla tiende a asegurar mayor atención, cuando asiente y sonríe mientras expresa al gunas 
de sus ideas.

Escucha activa: para asegurar que una conversa ción cumpla su propósito, es fundamental que quien escucha, evite cualquier tipo de distractor, de manera que su nivel de atención se mantenga a plenitud, esto, además de ser un acto de respeto, optimiza el tiempo y garantiza una mejor certeza en el flujo de ideas.

Cabe agregar que la asertividad es actualmente reconocida como una de las habilidades sociales más representativas y que su desempeño puede ser incrementado notablemente, teniendo en cuenta que, así como todas las habilidades físicas se desarrollan en la medida en que se permita al individuo expresarse motrizmente, también las habilidades sociales se fortalecen al ser entrenadas, sin embargo, es muy fácil encontrar un entrenador para fortalecer determinada habilidad motriz, lo que no ocurre, si se intenta buscar uno que ayude a desar rollar habilidades sociales, esto no significa que no existan, solo que los que hay, se encuentran en proporciones muy diferentes respecto a los primeros.

Siendo la anterior, una de las razones por las cuales recaen en los educadores el compromiso moral y social de emprender estrategias y posibilitar escenarios para el fomento de las habilidades sociales, es así, que en diferentes países muchos de los profesores a través de los campamentos educativo vienen generando un complemento en la formación de dimensiones y habilidades de los estudiantes, que ni ellos esperaban, fortaleciendo su autoestima mediante la potenciación de nuevas habilidades, aprendizajes al tiempo que aumenta su apetito cognitivo, lo que es imprescindible para todo aprendizaje significativo posible (Fuenteal ba et al., 2017).

En este sentido, se puede reconocer de la práctica de campamentos educativos y su relación con las habilidades sociales, un panorama apropiado para la consolidación de una futura línea de investigación.

\section{Conclusiones}

La primera conclusión permite develar que las ha bilidades sociales como riqueza educativa promovida en las instituciones escolares de carácter público del municipio deVillavicencio - Colombia, donde se vienen desarrollando prácticas de campamentos educativos; pueden convertirse en modelo para que otras instituciones incorporen este tipo de procesos teniendo en cuenta que el municipio cuenta con una variedad de lugares ideales para tal fin, como fincas agro-turísticas, escena rios naturales, clubes etc. En los que el acceso y la segu- ridad no son inconveniente. Por lo tanto, surge la necesidad de promocionar los campamentos educativos en las diferentes instituciones a través de las asociaciones de padres de familia y en la secretaría de educación municipal.

De acuerdo con las observaciones realizadas en lo avanzado del ejercicio investigativo, los campamentos educativos que se diseñan con el propósito de promover habilidades sociales en los escolares del municipio deVillavicencio - M eta, hacen uso de los elementos propios que caracterizan la práctica de un campamento convencional (nudos, amarres, técnicas, fogatas, técnicas de orientación etc.), con el fin de generar situaciones idea les que permitan abrir espacios de reflexión en torno a las habilidades sociales, de manera que la experiencia vivida sea el mejor ejemplo para poner en contexto lo que se pretende ensañar.

Por otro parte, es relevante enfatizar en la responsabilidad de quienes emanan y promueven las directrices de la práctica de campamento, ya que ésta debe ser asumida acorde a los propósitos del mismo, especialmente, si el tema a desarrollar propende por el fortalecimiento de habilidades sociales. En este sentido, las expectativas mínimas de los acampantes y de sus padres de familia se relacionan con tener la confianza de que dicha actividad no solo garantizará cuidados en la integridad de los participantes, sino que será coordinada por una persona amable, de buena empatía, solidaria, hábil frente a la resolución de conflictos, en fin, comprometida con el desarrollo de habilidades sociales.

Los campamentos educativos desarrollados en las instituciones escolares del municipio deVillavicencio Colombia, tienden a permitir que mediante un acto democrático los acampantes tengan la opción de elegir sus propios dirigentes, tal como el ciudadano elige a sus gobernantes, esto, puede funcionar como valor agrega do en el proceso de formación de ciudadanos, ya que analógicamente todo se podrá desarrollar a una pequeña escala enmarcada dentro de la gobernabilidad y gobernanza.

Finalmente, es necesario señalar que los campamentos educativos desarrollados por las instituciones escolares del municipio de Villavicencio - Colombia, conducentes al fortalecimiento de habilidades sociales tienen propensión a contribuir a la formación integral de los participantes, lo cual, ofrecerá ganancias que en términos de aprendizajes que se extrapolarán en cualquier contexto social alo largo de su vida; lo que significa, que este tipo de actividades favorece la proliferación de entornos de convivencia. 


\section{Referencias}

Alejandro, M. C. (2013). Lademocraciacomo políticapública: oportunidades para el fortalecimiento democrático. Revista de Estudios Sociales, (47), 25-38. Recuperado de: https:/ / revistas. uniandes. edu.co/ doi/ abs/10.7440/ res47.2013.02

Álvarez, R. L., \&Verrastro,V. (2013). Campamentos educativos para el siglo XXI: percepciones de referentes de establecimientoseinstituciones educativas en U ruguay. Reviisa U niverstaria delaEducación Físca y el Deporte, (6), 25-35. Recuperado de: http:/ / www.revistasiuacj.edu.uy/ index. php/ rev1/ article/ view/ 54 Caballo,V. E., \& Salazar, I. C. (2017). Desarrollo y validación de un nuevo instrumento paralaevaluación de las habilidades socia les: el» cuestionario de habilidades sociales»(CHASO). Behavioral Psychology/ Pscología Conducual, 25(1). Recupera do de: https:// n9.cl/ gl2d

Castañeda, L. (2011) Campamento. Armenia, Colombia: Editorial Kinesis.

CO LDEPO RTES. (2015). Programa N acional de Campamentos Juveniles. Bogotá, Colombia.

Ferrer, M. E. L., \& Sánchez, K. R. (2011). El campamento como programadidáctico: haciaunapropuestateórico-metodológica parasu implementación en losmuseos/ The camp asadidactic program: a theoretical and methodological proposal to the implementation in museums. Actualidades Investigativas en Educación, 11(1). Recuperado de: https:/ / revistas.ucr.ac.cr/ index. php/ aie/ article/ view / 10184

Fuenteal baCruz, M., M arín Isamit, F., Castillo Retamal, F., \& Roco Fuentes, L. (2017).Análisisdelaexperienciapedagógica: campamento EXPLORA Chile VA! Valorando la biodiversidad maulina. Actualidades Investigativas en Educación, 17(1), 229254. Recuperado de: https:// www. scielo.sa.cr/ s c i e I o.ph p ? p i d = S 1409 47032017000100229\&script=sci_arttext

Gómez,A., Gonnet, A., \& Lema, R. (2017). Fundamentosdelos campamentoseducativos: análisisdelaprimer Consultasobre Campamentos realizada por la Federación Sudamericana de Asociaciones Cristianas de Jóvenes (1961). Revista Universitaria de la Educación Físca y el Deporte, (10), 24-37. Recuperado de: http:/ / revistasiuacj. edu. uy/ index. php/ rev1/ article/ view/ 109

Mendoza, J. (2017). El Trabajo Colaborativo en la Sociedad del Conocimiento: unA nálisis Documental. Universidad deQ uintana Roo. Recuperado de: https:/ / n9. cl/ 4f3uk

Moyano, A. C., Pacheco, M. M., \& U rbieta, M. D. C. T. (2018). Procesospsicosocialesen Educación Física: actitudes, estrategias y clima motivacional percibido. Retos, nuevas tendencias en educación física, deporte y recreación, (34), 19-24. Recuperado de: https:/ / dialnet.unirioja. es/ servlet/ articulo?codigo $=6736361$

Pegal ajar, M. C. (2018). Formación en competenciasen alumnado universitario de Educación Social mediante prácticas basadas en aprendizaje cooperativo. Revista Complutense de Educación, 29(3), 829-845. Recuperado de: https:/ / revistas.ucm.es/ index. php/ RCED / article/ view/ 53970
Pereira, M. L. N. (2008). Relaciones interpersonales adecuadas mediante una comunicación y conducta asertivas/ Adequate human relationships through an assertive conducts and communication. Actualidades investigativas en educación, 8(1). Recuperado de: https./ / revistas. ucr.ac.cr/ index. php/ aie/ article/ view/ 9315

Pérez, I. P. (2000). Habilidadessociales educar hacia la autorregulacion: concepctualizacion, evaluacion e intervencion (Vol. 32). Barcelona, España: Horsori Editorial, Sl.

Ramos, Y. C., Cantillo, A. F., \& Bestard, C. M. (2014). Algunas consideraciones teóricas sobre las habilidades sociales. Revista Información Científica, 87(5), 949-959. Recuperado de: https:/ / www. redalyc. org/ pdf/ 5517/ 551757255019. pdf

Rodríguez, G.A. R., Hernandez, L. \& \&Triana, N.V. (2019).Valida ción de un cuestionario para evaluar valores personales y sociales en jóvenes deportistas. Retos, nuevas tendencias en educación físca, deportey recreación, (36), 152-158. Recuperado de: https:/ / recyt. fecyt.es/ index. php/ retos/ article/ view/ 65253

Rosales, K. N. V. (2017). Gestión de campamentos organizados para niños y jóvenes en México: formación y sustentabilidad a largo plazo. (Tesis). Universidad deQ uintana Roo. Recuperado de: http:/ / www. cozumel. uqroo. mx/ mgst/ productos/ Tesis_KNVR_Campamentos_M Mexico.pdf

SáezSotomajor, D., FigueroaM ayorga, 0. \& PereiraK ahl, S. (2018). Habilidades para la formación ciudadana, necesidades de la sociedad red en un mundo globalizado: Evaluación diagnóstica. Revista Boletín Redipe, 7(1), 62-69. Recuperado a partir de https: / / revista redipe.org/ index. php/ 1/ article/ view/ 417 Sánchez, S. (2016). Habilidades sociales. Madrid, España: Editorial Síntesis, S.A.

Tapia-Gutiérrez, Carmen Paz \& Cubo-Delgado, Sixto (2017). Habilidades sociales relevantes: percepciones de múltiples actores educativos. magis, Revista Internacional de Investigación en Educación, 9 (19), 133-148. Recuperado de: https:/ / revistas.javeriana. edu. co/ index. php/ MAGIS/ article/ view/ 18993

Vila, G. O., Rodríguez, J. R., Robles, M.T.A., González, L. J. D., Franco, J., Sánchez, A. C. J., \& Fuentes-Guerra, F. J. G. (2019). Las preferencias de interacción social en las Escuelas Sociodeportivas de Baloncesto de la Fundación Real Madrid. Retos. nuevas tendencias en educación física, deporte y recreación, (35), 101-106. Recuperado de: https:// recyt.fecyt.es/ index. php/ retos/ article/ view/ 62992

Vivas, M. M., Gómez, J. G., Bartoll, O. C., \& Miravet, L. M. (2017). Validación de una fichade observación parael anáisis de habilidades socio-emocionales en Educación Física. Retos. nuevas tendencias en educación física, deporte y recreación, (31), 8-13. Recuperado de: https: / / recyt. fecyt.es/ index. php/ retos/ article/ view/ 43815

Zipitría G. (2016). Teoría y organización del campamento educativo: apuntes del curso decampamento dictado en el IUAC). Montevideo, U ruguay: Instituto U niversitarioA sociación Cristianade óvenes. 